\title{
Noncausal Optimal Tracking of Linear Switched Systems
}

\author{
Gou Nakura \\ Osaka University, Department of Engineering \\ 2-1, Yamadaoka, Suita, Osaka, 565-0871, Japan \\ nakura@watt.mech.eng.osaka-u.ac.jp
}

\begin{abstract}
In this paper we consider the noncausal optimal tracking problem on the finite time interval for linear switched systems. We consider the problem to obtain the solution of both optimal switching sequences and optimal control inputs such that the tracking error is minimized. In this paper we assume that information of reference signals is known a priori for the whole time interval and utilize its information so that the tracking performace becomes better. We study a computation method of the optimal performance including some information of tracking errors and present an iterative algorithm to determine the optimal timing and optimal tracking performance numerically.
\end{abstract}

Key words: Switched systems; Optimal control; GSLQ problems; Noncausal tracking theory; Riccati equations

\section{Introduction}

On optimal control problems for switched systems, the problem to obtain the solution of both the optimal switching sequences and the optimal inputs is very important, and so much works have been done by many researchers recently $([1$, $2,6,19,20,22])$.

In particular, X. Xu and P. J. Antsaklis have studied the optimal timing and control problem by the parametrization approach([19,22]). They have decomposed the problem into two stages. In the first stage, they have considered a cost optimization problem over fixed switching sequences. In the second stage, they have considered a nonlinear optimization problem to find local switching sequences. In order to solve these two problems, they have presented an algorithm based on the gradient projection method and its variations([3]). For linear qudratic (LQ) problem, they have constructed the optimization algorithm by using the general Riccati equation parametrized by switching instants. The embedded control system theory is a more general control theory than the theory by their time parametrization approach for the switched systems. The switched systems can be "embedded" into a larger class of systems. Recently the relationship between the switched and embedded systems has been researched([2]). 
It is well known that, for design of tracking control systems, preview information of reference signals is very useful for improving the performance of the closed-loop systems, and much work has been done for preview control systems $([4,5,7-18])$. U. Shaked and C. E. de Souza have presented the $\mathrm{H}_{\infty}$ tracking theory with preview by a game theoretic approach([17]). Their theory can be restricted to optimal tracking theory and also extended to robust $\mathrm{H}_{\infty}$ tracking control theory $([18])$ or stochastic $\mathrm{H}_{\infty}$ tracking control theory([7-9]). Their theory has been applied to various types of systems, for example, continuous-time systems $([8,17,18])$, discrete-time systems $([4,7])$, impulsive systems $([13-16])$ and so on. In this paper we describe that their tracking theory can be applied to the switched systems.

There exist two structures on preview information. One is the fixed preview type that information of reference signals is known until fixed preview time length ahead. The other is the perfectly noncausal type that information of reference signals is known a priori for the whole time interval. In this paper we assume that the structure of the preview information is the perfectly noncausal type.

In this paper we study the noncausal optimal timing and tracking control problem for linear switched systems based on the time parametrization approach by X. Xu and P. J. Antsaklis([19,22]). In order to design noncausal feedforward compensators, we consider a vector and its dynamics introducing future information of reference signals([17]). As the cases of the previous various preview or noncausal tracking control theory $([4,5,7-18])$, we can expect improvement of tracking performance by introducing future information. We present a practical optimization algorithm, which is an extension of the time parametrization method by X. Xu and P. J. Antsaklis([19,22]). Their algorithm is feasible for numerical computation in the sense of not demanding more than solving a set of ODEs (ordinary differential equations) with boundary conditions. Compared with it, our algorithm on the tracking problem needs some numerical integration to add to the task of solving the set of ODEs.

The organization of this paper is as follows. In section 2 we describe our systems and problem formulation. In section 3 we present the equivalent noncausal GSLQ(General Switched Linear Quadratic) tracking problem and, under the assumption of the fixed switching instants, give the necessary and sufficient conditions of the solvability and a control strategy for this problem. In section 4 we construct the optimization algorithm of both switching instants and tracking performance, based on the theory described in the section 3. In the appendix we describe the proof of Proposition 1, which gives the necessary and sufficient conditions of the solvability and a control strategy for the equivalent noncausal GSLQ tracking problem under the assumption of the fixed switching instants.

Notations: Throughout this paper the subscript "' stands for the matrix transposition, $\|\cdot\|$ denotes the Euclidian vector norm and $\|v\|_{R}^{2}$ also denotes the weighted norm $v^{\prime} R v$. 


\section{Problem Formulation}

Consider the following switched systems with effects of reference signal.

$$
\begin{aligned}
& \dot{x}(t)=A_{1} x(t)+B_{2,1} u(t)+B_{3,1} r_{c}(t), t_{0} \leq t<t_{1}, x\left(t_{0}\right)=x_{0}, \\
& \dot{x}(t)=A_{2} x(t)+B_{2,2} u(t)+B_{3,2} r_{c}(t), t_{1} \leq t \leq T \\
& z_{c}(t)=C_{1} x(t)+D_{12} u(t)+D_{13} r_{c}(t)
\end{aligned}
$$

where $x \in \mathbf{R}^{\mathbf{n}}$ is the state, $u \in \mathbf{R}^{\mathbf{m}}$ is the control input, $z_{c} \in \mathbf{R}^{\mathbf{k}_{\mathbf{c}}}$ is the controlled output, $r_{c}(t) \in \mathbf{R}^{\mathbf{r}_{\mathbf{c}}}$ is an known or mesurable reference signal. $x_{0}$ is a given initial state. $t_{0}$ and $T$ is given initial and terminal times, and $t_{1}$ is a switching time to be sought such that the tracking performance becomes optimal. We assume that all system matrices are constant and of compatible dimensions.

Remark 1. The generalized system with $K$ switching instants of the system (1) can be represented as follows:

$$
\begin{gathered}
\dot{x}(t)=A_{1} x(t)+B_{2,1} u(t)+B_{3,1} r_{c}(t), t_{0} \leq t<t_{1}, x\left(t_{0}\right)=x_{0}, \\
\dot{x}(t)=A_{2} x(t)+B_{2,2} u(t)+B_{3,2} r_{c}(t), t_{1} \leq t<t_{2} \\
\quad \ldots \\
\dot{x}(t)=A_{K+1} x(t)+B_{2, K+1} u(t)+B_{3, K+1} r_{c}(t), t_{K} \leq t \leq T
\end{gathered}
$$

Throughout this paper, for simplification, we mainly consider the system (1), which has only one switching instant.

For the system (1), we assume the following condition.

$$
\text { A1: } D_{12}^{\prime} D_{12}>O
$$

We define the following performance index for the system (1)

$$
J:=\int_{t_{0}}^{T}\left\|z_{c}(t)\right\|^{2} d t+x^{\prime}(T) Q_{f} x(T)
$$

where $Q_{f} \geq O$. Partially, in this paper, in order to clarify the switching intants, we denote $J$ as $J\left(t_{1}\right)$ and etc.

Remark 2. On defining the performance index, U. Shaked and C. E. de Souza([17]) have introduced the expectation $\mathbf{E}_{\bar{R}_{s}}$ considering the average of the performance index over the statistics of the unknown part $\bar{R}_{s+h}:=\left\{r_{c}(l) ; s+h<l \leq T\right\}$ of the reference signal $r_{c}$ where $h$ is a fixed preview length. Note that, compared with it, we do not introduce any expectation operators. As U. Shaked and C. E. de Souza have described in [17], the expectation operator is superfluous in the case of perfectly noncausal settings because we don't have to consider both of causal and noncausal parts on the whole time interval, i.e., we don't have to consider any unknown parts of reference signals at the current time on the whole time interval $\left[t_{0}, T\right]$. 
For the system (1) and the performance index (3), we consider the following noncausal optimization problem.

Noncausal Optimal Timing and Tracking Problem :

Consider the system (1) and the performance index (3), and assume the condition A1. Assume also that the reference signal $\left\{r_{c}(t)\right\}$ is known a priori for the whole time interval $t \in\left[t_{0}, T\right]$. Then, find $\left\{u^{*}\right\}$ and a switching instant $t_{1}$ minimizing the performance index $(3)$.

In order to solve this problem, we consider the following optimization algorithm as $[19,22]$. However, note that our performance index can include any noncausal information of tracking signals.

- Algorithm 1

1) Set the iteration index $j=0$. Choose an initial $t_{1}^{j}$.

2) By solving an optimal noncausal tracking problem, find $J\left(t_{1}^{j}\right)$.

3) Compute $\left(\partial J / \partial t_{1}\right)\left(t_{1}^{j}\right)$ (and $\left(\partial^{2} J / \partial t_{1}^{2}\right)$ if second-order method is to be used).

4) Use some feasile direction method to update to be $t_{1}^{j+1}=t_{1}^{j}+\alpha^{j}\left(\partial J / \partial t_{1}\right)\left(t_{1}^{j}\right)$ (the stepsize $\alpha^{j}$ can be chosen using some stepsize rule, e.g.,Armijo's rule([3])). Set the iteration index $j=j+1$.

5) Repeat Steps 2), 3), 4) and 5), until, for a given small number $\epsilon>0$, $\left|\partial J / \partial t_{1}\right|<\epsilon$.

How can we compute this gradient of the performance index? X. Xu and P. J. Antsaklis $([19,22])$ have presented how to compute the gradient for the GSLQ problem using the generalized Riccati equation denpendent on the parameter, but not considering any exogeneous tracking signals nor noncausal information. We present an algorithm including how to compute the gradient with the tracking error, considering the effects of the exogeneous and noncausal tracking error over the whole time interval $[0, T]$. In order to construct the algorithm, we utilize the dynamics which gives the noncausal information of the tracking signal with the parametrized general Riccati equation by $\mathrm{X}$. Xu and P. J. Antsaklis together.

\section{Approach Based on The Parametrization of The Switching Instants}

In order to realize the algorithm for the Noncausal Optimal Timing and Tracking Problem in the previous section, we take the following steps.

Step 1: We parameterized and reduce our problem to the equivalent GSLQ Tracking Problem.

Step 2: We seek the solution of the equivalent GSLQ Tracking Problem for the fixed $x_{n+1}$.

Step 3: Minimize $J$ with respect to varying $x_{n+1}$.

First we introduce a state variable $x_{n+1}$ corresponding to the switching instant $t_{1}$. Let $x_{n+1}$ satisfy

$$
\frac{d x_{n+1}}{d t}=0, x_{n+1}(0)=t_{1}
$$


Moreover, we introduce a new independent time variable $\tau$ and define a piecewise linear relationship between $t$ and $\tau$ as follows:

$$
t=\left\{\begin{array}{cc}
t_{0}+\left(x_{n+1}-t_{0}\right) \tau, & 0 \leq \tau \leq 1 \\
x_{n+1}+\left(T-x_{n+1}\right)(\tau-1) & 1 \leq \tau \leq 2 .
\end{array}\right.
$$

By this parametrization of the switching time, our Noncausal Optimal Timing and Tracking Problem is transcribed into the following equivalent problem.

\section{Equivalent Noncausal GSLQ (General Switched Linear Quadratic)} Tracking Problem

Consider the system

$$
\frac{d x(\tau)}{d \tau}=\left(x_{n+1}-t_{0}\right)\left(A_{1} x(\tau)+B_{2,1} u(\tau)+B_{3,1} r_{c}(\tau)\right), \frac{d x_{n+1}(\tau)}{d \tau}=0
$$

for $\tau \in[0,1)$ and

$$
\frac{d x(\tau)}{d \tau}=\left(T-x_{n+1}\right)\left(A_{2} x(\tau)+B_{2,2} u(\tau)+B_{3,2} r_{c}(\tau)\right), \frac{d x_{n+1}(\tau)}{d \tau}=0
$$

for $\tau \in[1,2] . t_{0}, T$ and $x(0)$ are given. Assume that the reference signal $\left\{r_{c}(t)\right\}$ is known a priori for the whole time interval $\tau \in[0,2]$. Find an $x_{n+1}$ and a $u(\tau)$ such that the parametrized performance index

$$
J=x^{\prime}(2) Q_{f} x(2)+\int_{0}^{1}\left(x_{n+1}-t_{0}\right)\left\|z_{c}(\tau)\right\|^{2} d \tau+\int_{1}^{2}\left(T-x_{n+1}\right)\left\|z_{c}(\tau)\right\|^{2} d \tau
$$

is minimized.

Note that this problem no longer includes any varying switching instants. However, it is difficult to solve this problem because of the nonlinearity of the whole system including the variable $x_{n+1}$. In order to solve this problem, first we assume that $x_{n+1}$ is fixed. Now we consider the following general Riccati equation and terminal condition parametrized by $x_{n+1}$.

$$
\begin{aligned}
& -\frac{\partial X}{\partial \tau}=\left(x_{n+1}-t_{0}\right)\left(A_{1}^{\prime} X+X A_{1}+C_{1}^{\prime} C_{1}-\tilde{S}_{1}^{\prime} \tilde{R}^{-1} \tilde{S}_{1}\right) \text { for } \tau \in[0,1), \\
& -\frac{\partial X}{\partial \tau}=\left(T-x_{n+1}\right)\left(A_{2}^{\prime} X+X A_{2}+C_{1}^{\prime} C_{1}-\tilde{S}_{2}^{\prime} \tilde{R}^{-1} \tilde{S}_{2}\right) \text { for } \tau \in[1,2]
\end{aligned}
$$

and $X\left(2, x_{n+1}\right)=Q_{f}$ where

$$
\tilde{R}=D_{12}^{\prime} D_{12}, \tilde{S}_{1}(\tau)=B_{2,1}^{\prime} X(\tau)+D_{12}^{\prime} C_{1}, \tilde{S}_{2}(\tau)=B_{2,2}^{\prime} X(\tau)+D_{12}^{\prime} C_{1} .
$$

Remark 3. This type of Riccati equation is the same as the one for the standard GSLQ problem by $[19,22]$ not considering the effects of any reference signals nor noncausal information. 
Then we have the following proposition, which gives the solvability and an optimal control strategy for our equivalent GSLQ tracking problem under the assumption of the fixed $x_{n+1}$. This proposition is an extension of the noncausal tracking control theory by U. Shaked and C. E. de Souza([17]).

Proposition 1. Consider the system (5)-(6) and the performance index (7), and suppose A1. Assume that $x_{n+1}$ is fixed. Then the equivalent GSLQ tracking problem is solvable by state feedback if and only if there exists a matrix $X\left(\tau, x_{n+1}\right)$ satisfying the conditions $X\left(\tau, x_{n+1}\right)=X^{\prime}\left(\tau, x_{n+1}\right)$ and $X\left(2, x_{n+1}\right)=$ $Q_{f}$ such that the equation (8)-(9) holds over $\tau \in[0,2]$. Then an optimal control strategy for our noncausal tracking problem (5)-(6) and (7) is given by

$$
\begin{aligned}
& u_{c, 1}^{*}\left(\tau, x_{n+1}\right)=-\tilde{R}^{-1} \tilde{S}_{1} x\left(\tau, x_{n+1}\right) \\
&-\mathbf{C}_{u} r_{c}\left(\tau, x_{n+1}\right)-\mathbf{C}_{\theta u, 1} \theta\left(\tau, x_{n+1}\right) \text { for } 0 \leq \tau<1, \\
& u_{c, 2}^{*}\left(\tau, x_{n+1}\right)=-\tilde{R}^{-1} \tilde{S}_{2} x\left(\tau, x_{n+1}\right) \\
&-\mathbf{C}_{u} r_{c}\left(\tau, x_{n+1}\right)-\mathbf{C}_{\theta u, 2} \theta\left(\tau, x_{n+1}\right) \text { for } 1 \leq \tau \leq 2
\end{aligned}
$$

where

$$
\mathbf{C}_{\theta u, 1}=\tilde{R}^{-1} B_{2,1}^{\prime}, \mathbf{C}_{\theta u, 2}=\tilde{R}^{-1} B_{2,2}^{\prime}, \mathbf{C}_{u}=\tilde{R}^{-1} D_{12}^{\prime} D_{13} .
$$

$\theta(t), t \in[0, T]$, satisfies the dynamics

$$
\begin{aligned}
\frac{\partial}{\partial \tau} \theta\left(\tau, x_{n+1}\right)=\left(x_{n+1}-t_{0}\right)\left\{-\bar{A}_{c, 1}^{\prime}(\right. & \left(x_{n+1}\right) \theta\left(\tau, x_{n+1}\right) \\
& \left.+\bar{B}_{c, 1}\left(\tau, x_{n+1}\right) r_{c}\left(\tau, x_{n+1}\right)\right\}, 0 \leq \tau<1, \\
\frac{\partial}{\partial \tau} \theta\left(\tau, x_{n+1}\right)=\left(T-x_{n+1}\right)\left\{-\bar{A}_{c, 2}^{\prime}(\right. & \left(\tau, x_{n+1}\right) \theta\left(\tau, x_{n+1}\right) \\
+ & \left.\bar{B}_{c, 2}\left(\tau, x_{n+1}\right) r_{c}\left(\tau, x_{n+1}\right)\right\}, 1 \leq \tau \leq 2
\end{aligned}
$$

and the terminal condition $\theta\left(2, x_{n+1}\right)=0$ where

$$
\begin{aligned}
& \bar{A}_{c, i}\left(\tau, x_{n+1}\right)=A_{i}-B_{2, i} \tilde{R}^{-1} \tilde{S}_{i}\left(\tau, x_{n+1}\right), \\
& \bar{B}_{c, i}\left(\tau, x_{n+1}\right)=-\left(X\left(\tau, x_{n+1}\right) B_{3, i}+C_{1}^{\prime} D_{13}\right)+\tilde{S}_{i}^{\prime}\left(\tau, x_{n+1}\right) \mathbf{C}_{u} \text { for } i=1,2 .
\end{aligned}
$$

Moreover, the parametrized value at $\tau=0$ of performance index by the optimal input $u_{c, i}^{*}$ for $i=1,2$ is

$$
\begin{gathered}
J\left(t_{1}\right)=J\left(x_{n+1}\right)=V\left(x_{0}, 0, x_{n+1}\right) \\
=x_{0}^{\prime} X\left(0, x_{n+1}\right) x_{0}+2 \theta^{\prime}\left(0, x_{n+1}\right) x_{0}+\left(x_{n+1}-t_{0}\right) \int_{0}^{1} \delta \bar{J}_{c, 1}\left(r_{c}, \tau, x_{n+1}\right) d \tau \\
+\left(T-x_{n+1}\right) \int_{1}^{2} \delta \bar{J}_{c, 2}\left(r_{c}, \tau, x_{n+1}\right) d \tau
\end{gathered}
$$

where

$$
\begin{aligned}
& \delta \bar{J}_{c, i}\left(r_{c}, \tau, x_{n+1}\right)=\delta J_{c}\left(r_{c}, \tau, x_{n+1}\right) \\
& +2 \theta^{\prime}\left(\tau, x_{n+1}\right) B_{3, i} r_{c}\left(\tau, x_{n+1}\right) \\
& -2 \theta^{\prime}\left(\tau, x_{n+1}\right) \mathbf{C}_{\theta u, i}^{\prime} \tilde{R}^{\prime} \mathbf{C}_{u} r_{c}\left(\tau, x_{n+1}\right)-\left\|\tilde{R}^{1 / 2} \mathbf{C}_{\theta u, i} \theta\left(\tau, x_{n+1}\right)\right\|^{2}, i=1,2
\end{aligned}
$$


and

$$
\delta J_{c}\left(r_{c}, \tau, x_{n+1}\right)=\left\|D_{13} r_{c}\left(\tau, x_{n+1}\right)\right\|^{2}-\left\|\tilde{R}^{1 / 2} \mathbf{C}_{u} r_{c}\left(\tau, x_{n+1}\right)\right\|^{2} .
$$

(Proof): See appendix.

Remark 4. In the case of the causal tracking problem, in which we do not consider any future information of tracking signals, the control strategy is

$$
\begin{aligned}
& u_{c, 1}^{*}\left(\tau, x_{n+1}\right)=-\tilde{R}^{-1} \tilde{S}_{1} x\left(\tau, x_{n+1}\right)-\mathbf{C}_{u} r_{c}\left(\tau, x_{n+1}\right) \text { for } 0 \leq \tau<1, \\
& u_{c, 2}^{*}\left(\tau, x_{n+1}\right)=-\tilde{R}^{-1} \tilde{S}_{2} x\left(\tau, x_{n+1}\right)-\mathbf{C}_{u} r_{c}\left(\tau, x_{n+1}\right) \text { for } 1 \leq \tau \leq 2,
\end{aligned}
$$

using the solution of the Riccati equation (8)-(9), and the parametrized value at $\tau=0$ of performance index by the optimal input $u_{c, i}^{*}$ for $i=1,2$ is

$$
\begin{aligned}
& J\left(t_{1}\right)=J\left(x_{n+1}\right)=V\left(x_{0}, 0, x_{n+1}\right) \\
& =x_{0}^{\prime} X\left(0, x_{n+1}\right) x_{0}+\left(x_{n+1}-t_{0}\right) \int_{0}^{1} \delta J_{c}\left(r_{c}, \tau, x_{n+1}\right) d \tau \\
& +\left(T-x_{n+1}\right) \int_{1}^{2} \delta J_{c}\left(r_{c}, \tau, x_{n+1}\right) d \tau .
\end{aligned}
$$

By comparing the value of performance index in the case of the noncausal tracking with the one in the case of the causal numerically, we can quantitatively verify whether or not the tracking performance becomes better by the noncausal information of the tracking signal.

We can obtain the value of $J\left(x_{n+1}\right)$ at $\tau=0$ by solving (8)-(9) and (12)-(13) (for a fixed $\left.x_{n+1}\right)$ backward in $\tau$ along with the terminal conditions $X\left(T, x_{n+1}\right)=$ $Q_{f}$ and $\theta\left(T, x_{n+1}\right)=0$. Moreover how can we numerically calculate the gradient $d J\left(x_{n+1}\right) / d x_{n+1}$ ? We will describe the method of calculus of the gradient $d J\left(x_{n+1}\right) / d x_{n+1}$ in the next section.

\section{Construction of An Algorithm for Numerical Computation}

Our issue is to find the optimal point $x_{n+1}$ at which the performance index including the tracking error is minimized. For this purpose, we need calculate the gradient $d J\left(x_{n+1}\right) / d x_{n+1}$.

Hence, throughout in this section, we assume the following condition for the reference signal $r_{c}(t)$.

A2: $r_{c}$ is at least $C^{2}$ class function.

We need calculate the gradient $d J\left(x_{n+1}\right) / d x_{n+1}$ according to the optimization algorithm. From (14), we obtain

$$
\frac{d J\left(x_{n+1}\right)}{d x_{n+1}}=x_{0}^{\prime} \frac{\partial X\left(0, x_{n+1}\right)}{\partial x_{n+1}} x_{0}+2\left(\frac{\partial \theta^{\prime}\left(0, x_{n+1}\right)}{\partial x_{n+1}}\right) x_{0}
$$




$$
\begin{gathered}
+\int_{0}^{1} \delta \bar{J}_{c, 1}\left(r_{c}, \tau, x_{n+1}\right) d \tau-\int_{1}^{2} \delta \bar{J}_{c, 2}\left(r_{c}, \tau, x_{n+1}\right) d \tau \\
+\left(x_{n+1}-t_{0}\right) \int_{0}^{1} \frac{\partial \delta \bar{J}_{c, 1}}{\partial x_{n+1}}\left(r_{c}, \tau, x_{n+1}\right) d \tau \\
+\left(T-x_{n+1}\right) \int_{1}^{2} \frac{\partial \delta \bar{J}_{c, 2}}{\partial x_{n+1}}\left(r_{c}, \tau, x_{n+1}\right) d \tau .
\end{gathered}
$$

In order to obtain the value at $x_{n+1}$ of this right hand side, we make calculus as follows.

By differentiatting (8) and (9), we have the following equation.

$$
\begin{aligned}
-\frac{\partial}{\partial \tau}\left(\frac{\partial X}{\partial x_{n+1}}\right)= & A_{1}^{\prime} X+X A_{1}+C_{1}^{\prime} C_{1}-\tilde{S}_{1}^{\prime} \tilde{R}^{-1} \tilde{S}_{1} \\
+\left(x_{n+1}-t_{0}\right) & \left(\frac{\partial X}{\partial x_{n+1}} A_{1}+A_{1}^{\prime} \frac{\partial X}{\partial x_{n+1}}\right. \\
& -\left(B_{2,1}^{\prime} \frac{\partial X}{\partial x_{n+1}}+D_{12}^{\prime} C_{1}\right)^{\prime} \tilde{R}^{-1}\left(B_{2,1}^{\prime} X+D_{12}^{\prime} C_{1}\right) \\
& \left.-\left(B_{2,1}^{\prime} X+D_{12}^{\prime} C_{1}\right)^{\prime} \tilde{R}^{-1}\left(B_{2,1}^{\prime} \frac{\partial X}{\partial x_{n+1}}+D_{12}^{\prime} C_{1}\right)\right)
\end{aligned}
$$

over the time interval $\tau \in[0,1)$ and

$$
\begin{aligned}
-\frac{\partial}{\partial \tau}\left(\frac{\partial X}{\partial x_{n+1}}\right)= & -\left(A_{2}^{\prime} X+X A_{2}+C_{1}^{\prime} C_{1}-\tilde{S}_{2}^{\prime} \tilde{R}^{-1} \tilde{S}_{2}\right) \\
+\left(T-x_{n+1}\right) & \left(\frac{\partial X}{\partial x_{n+1}} A_{2}+A_{2}^{\prime} \frac{\partial X}{\partial x_{n+1}}\right. \\
& -\left(B_{2,2}^{\prime} \frac{\partial X}{\partial x_{n+1}}+D_{12}^{\prime} C_{1}\right)^{\prime} \tilde{R}^{-1}\left(B_{2,2}^{\prime} X+D_{12}^{\prime} C_{1}\right) \\
& \left.-\left(B_{2,2}^{\prime} X+D_{12}^{\prime} C_{1}\right)^{\prime} \tilde{R}^{-1}\left(B_{2,2}^{\prime} \frac{\partial X}{\partial x_{n+1}}+D_{12}^{\prime} C_{1}\right)\right)
\end{aligned}
$$

over the time interval $\tau \in[1,2]$. Note that $\left(\partial / \partial x_{n+1}\right)(\partial / \partial \tau)=(\partial / \partial \tau)\left(\partial / \partial x_{n+1}\right)$ and we have used the general Riccati equation (8)-(9) on these calculus.

Moreover, with respect to the terms including the tracking errors, we have

$$
\begin{aligned}
& \frac{\partial \delta \bar{J}_{c, i}}{\partial x_{n+1}}=\frac{\partial \delta J_{c}}{\partial x_{n+1}} \\
& \quad+2\left(\frac{\partial \theta}{\partial x_{n+1}}\right)^{\prime} B_{3, i} r_{c}\left(\tau, x_{n+1}\right)+2 \theta^{\prime}\left(\tau, x_{n+1}\right) B_{3, i} \frac{\partial r_{c}}{\partial x_{n+1}} \\
& -\left\{2\left(\frac{\partial \theta}{\partial x_{n+1}}\right)^{\prime} \mathbf{C}_{\theta u, i}^{\prime} \tilde{R}^{\prime} \mathbf{C}_{u} r_{c}\left(\tau, x_{n+1}\right)+2 \theta^{\prime}\left(\tau, x_{n+1}\right) \mathbf{C}_{\theta u, i}^{\prime} \tilde{R}^{\prime} \mathbf{C}_{u} \frac{\partial r_{c}}{\partial x_{n+1}}\right\} \\
& -2 \theta^{\prime}\left(\tau, x_{n+1}\right) \mathbf{C}_{\theta u, i}^{\prime} \tilde{R} \mathbf{C}_{\theta u, i} \frac{\partial \theta}{\partial x_{n+1}}, i=1,2
\end{aligned}
$$


and

$$
\frac{\partial \delta J_{c}}{\partial x_{n+1}}=2 r_{c}^{\prime}\left(\tau, x_{n+1}\right) D_{13}^{\prime} D_{13} \frac{\partial r_{c}}{\partial x_{n+1}}-2 r_{c}^{\prime}\left(\tau, x_{n+1}\right) \mathbf{C}_{u}^{\prime} \tilde{R} \mathbf{C}_{u} \frac{\partial r_{c}}{\partial x_{n+1}}
$$

Now we need the value of $\partial \theta / \partial x_{n+1}$ at $\tau \in[0,2]$. By differentiatting $\partial \theta / \partial x_{n+1}$ with respect to $\tau \in[0,2]$ and using the dynamics (12)-(13) of $\theta$, we obtain the following equality.

$$
\begin{aligned}
& \frac{\partial}{\partial \tau}\left(\frac{\partial \theta}{\partial x_{n+1}}\right)=\frac{\partial}{\partial x_{n+1}}\left(\frac{\partial \theta}{\partial \tau}\right) \\
= & -\bar{A}_{c, 1}^{\prime} \theta\left(\tau, x_{n+1}\right)-\left(x_{n+1}-t_{0}\right)\left\{\bar{A}_{c, 1}^{\prime} \frac{\partial \theta}{\partial x_{n+1}}+\frac{\partial \bar{A}_{c, 1}^{\prime}}{\partial x_{n+1}} \theta\left(\tau, x_{n+1}\right)\right\} \\
& +\bar{B}_{c, 1} r_{c}\left(\tau, x_{n+1}\right)+\left(x_{n+1}-t_{0}\right)\left\{\frac{\partial \bar{B}_{c, 1}}{\partial x_{n+1}} r_{c}\left(\tau, x_{n+1}\right)+\bar{B}_{c, 1} \frac{\partial r_{c}}{\partial x_{n+1}}\right\},
\end{aligned}
$$

for $\tau \in[0,1)$, where

$$
\begin{aligned}
\frac{\partial \bar{A}_{c, 1}}{\partial x_{n+1}} & =-B_{2,1} \tilde{R}^{-1} \frac{\partial \tilde{S}_{1}}{\partial x_{n+1}}=-B_{2,1} \tilde{R}^{-1} B_{2,1}^{\prime} \frac{\partial X}{\partial x_{n+1}} \\
\frac{\partial \bar{B}_{c, 1}}{\partial x_{n+1}} & =-\frac{\partial X}{\partial x_{n+1}} B_{3,1}+\frac{\partial X}{\partial x_{n+1}} B_{2,1} \mathbf{C}_{u} .
\end{aligned}
$$

Similarly, for $\tau \in[1,2]$,

$$
\begin{aligned}
& \frac{\partial}{\partial \tau}\left(\frac{\partial \theta}{\partial x_{n+1}}\right)=\frac{\partial}{\partial x_{n+1}}\left(\frac{\partial \theta}{\partial \tau}\right) \\
= & \bar{A}_{c, 2}^{\prime} \theta\left(\tau, x_{n+1}\right)-\left(T-x_{n+1}\right)\left\{\bar{A}_{c, 2}^{\prime} \frac{\partial \theta}{\partial x_{n+1}}+\frac{\partial \bar{A}_{c, 2}^{\prime}}{\partial x_{n+1}} \theta\left(\tau, x_{n+1}\right)\right\} \\
& -\bar{B}_{c, 2} r_{c}\left(\tau, x_{n+1}\right)+\left(T-x_{n+1}\right)\left\{\frac{\partial \bar{B}_{c, 2}}{\partial x_{n+1}} r_{c}\left(\tau, x_{n+1}\right)+\bar{B}_{c, 2} \frac{\partial r_{c}}{\partial x_{n+1}}\right\}
\end{aligned}
$$

where

$$
\begin{aligned}
\frac{\partial \bar{A}_{c, 2}}{\partial x_{n+1}} & =-B_{2,2} \tilde{R}^{-1} \frac{\partial \tilde{S}_{2}}{\partial x_{n+1}}=-B_{2,2} \tilde{R}^{-1} B_{2,2}^{\prime} \frac{\partial X}{\partial x_{n+1}} \\
\frac{\partial \bar{B}_{c, 2}}{\partial x_{n+1}} & =-\frac{\partial X}{\partial x_{n+1}} B_{3,2}+\frac{\partial X}{\partial x_{n+1}} B_{2,2} \mathbf{C}_{u} .
\end{aligned}
$$

Note that these equalities (8)-(9), (12)-(13), (19)-(20) and (21)-(22) are a set of ODEs for $X\left(\tau, x_{n+1}\right), \theta\left(\tau, x_{n+1}\right), \partial X / \partial x_{n+1}$ and $\partial \theta / \partial x_{n+1}$ with the boundary conditions

$$
X\left(2, x_{n+1}\right)=Q_{f}, \frac{\partial X}{\partial x_{n+1}}\left(2, x_{n+1}\right)=O, \theta\left(2, x_{n+1}\right)=0, \frac{\partial \theta}{\partial x_{n+1}}\left(2, x_{n+1}\right)=0
$$

at $\tau=2$.

Now we obtain the following modified algorithm to obtain the values of the gradients of the performance index and the optimal timing numerically. 
- Algorithm 2 (Modified Algorithm)

1) Set the iteration index $j=0$. Choose an initial $x_{n+1}^{j}\left(=t_{1}^{j}\right)$. (Then $\tau=1$.)

2) By solving an optimal noncausal tracking problem, find $J\left(x_{n+1}^{j}\right)$.

In order to compute $J\left(x_{n+1}^{j}\right)$ numerically,

2a) For the given $x_{n+1}^{j}$, solve (8)-(9) with $X\left(2, x_{n+1}\right)=O$.

$2 \mathrm{~b})$ For the given $x_{n+1}^{j}$, solve (12)-(13) with $\theta\left(2, x_{n+1}\right)=0$ based on the information of $r_{c}$ over the whole time interval $\tau \in[0,2]$.

2c) Collectting the values over $\tau \in[0,2]$ obtained in 2a) and 2b), we do a numerical integration to obtain the value of $J\left(x_{n+1}^{j}\right)$ (cf.(14) in the noncausal case and (17) in the causal case)

3) Compute $\left(\partial J / \partial x_{n+1}\right)\left(x_{n+1}^{j}\right)$ (and $\left(\partial^{2} J / \partial^{2} x_{n+1}\right)\left(x_{n+1}^{j}\right)$ if second-order method is to be used).

In order to compute $\left(\partial J / \partial x_{n+1}\right)\left(x_{n+1}^{j}\right)$ numerically,

$3 a)$ For the given $x_{n+1}^{j}$, solve (19)-(20) with $\partial X / \partial x_{n+1}\left(2, x_{n+1}\right)=O$ utilizing the result of $2 \mathrm{a}$ ).

$3 \mathrm{~b})$ For the given $x_{n+1}^{j}$, solve (21)-(22) with $\partial \theta / \partial x_{n+1}\left(2, x_{n+1}\right)=0$ utilizing the result of $2 \mathrm{a}$ ), 2b) and $3 \mathrm{a}$ )

$3 c)$ Collectting the values over $\tau \in[0,2]$ obtained in $2 \mathrm{a}), 2 \mathrm{~b}), 3 \mathrm{a}$ ) and $3 \mathrm{~b})$, we do a numerical integration to obtain the value of $\left(\partial J / \partial x_{n+1}\right)\left(x_{n+1}^{J}\right)$ (cf.(18)) Or, instead of 2) and 3),

2-3) For the given $x_{n+1}^{j}$, solve a set of ODEs (8)-(9), (12)-(13), (19)-(20) and (21)-(22) with $X\left(2, x_{n+1}\right)=O, \theta\left(2, x_{n+1}\right)=0, \partial X / \partial x_{n+1}\left(2, x_{n+1}\right)=O$ and $\partial \theta / \partial x_{n+1}\left(2, x_{n+1}\right)=0$ to obtain the value of $J\left(x_{n+1}^{j}\right)$ and $\left(\partial J / \partial x_{n+1}\right)\left(x_{n+1}^{j}\right)$ numerically. (cf.(14)(17)(18))

4) Use some feasile direction method to update to be $x_{n+1}^{j+1}=x_{n+1}^{j}+\alpha^{j}$ $\left(\partial J / \partial x_{n+1}\right)\left(x_{n+1}^{j}\right)$ (in the case of second-order method, $x_{n+1}^{j+1}=x_{n+1}^{j}-\alpha^{j}$ $\left(\partial^{2} J / \partial x_{n+1}^{2}\right)^{-1}\left(\partial J / \partial x_{n+1}\right)\left(x_{n+1}^{j}\right)$, e.g., refer to [21])

(the stepsize $\alpha^{j}$ can be chosen using some stepsize rule, e.g.,Armijo's rule[3]). Set the iteration index $j=j+1$.

5) Repeat Steps 2), 3), 4) and 5), until, for a given small number $\epsilon,\left|\partial J / \partial x_{n+1}\right|<$ $\epsilon$.

Remark 5. In order to obtain not only the initial value but also the value of the tracking error term numerically, we need not only the initial value but also the intermediate value of the solutions for the set of ODEs (8)-(9), (12)-(13), (19)-(20) and (21)-(22). For example, we set a sufficiently small sampling time and approximately calculate the value of the integral to obtain the values of the gradients $d J / d x_{n+1}$ at the switching instants.

Remark 6. Note that, as we have described in Remark 4, in the case of the causal tracking problem, we do not need any information with respect to the values of $\theta$ and $\partial \theta / \partial x_{n+1}$, which is different from the case of the noncausal tracking problem.

Remark 7. The Cases of More Than One Switching Times: It can be seen that there is no difficulty in applying the previous method to GSLQ tracking problems 
in the cases with more than one switchings as GSLQ problems not considering any tracking signals. In detail, we can construction the algorithm for the optimization as follows: Assuming that there exist $K$ switchings, we can transcribe the original optimal tracking problem into an equivalent problem by introducing $K$ new state variables $x_{n+i}, i=1, \cdots, K$ which correspond to the switching instants $t_{i}$ and satisfy

$$
\frac{d x_{n+1}}{d t}=0, x_{n+i}(0)=t_{i}
$$

The new independent time variable $\tau$ has the following piecewise linear relationship between $t$ and $\tau$.

$$
t=\left\{\begin{array}{cc}
t_{0}+\left(x_{n+1}-t_{0}\right) \tau, & 0 \leq \tau \leq 1 \\
x_{n+1}+\left(x_{n+2}-x_{n+1}\right)(\tau-1), & 1 \leq \tau \leq 2 \\
\cdots & \cdots \\
x_{n+K}+\left(T-x_{n+K}\right)(\tau-K), & K \leq \tau \leq K+1
\end{array}\right.
$$

Note that $\tau=0$ corresponds to $t=t_{0}, \tau=1$ corresponds $t=t_{1}, \ldots$, and $\tau=K+1$ corresponds to $t=T$. Then, differentiating the Riccati equations and the dynamics of $\theta$ parametrized by $x_{n+1}, \cdots, x_{n+K}$, we can obtain additional equations for $\partial X / \partial x_{n+i}$ and $\partial \theta / \partial x_{n+i}$. Along with the boundary conditions $X\left(K+1, x_{n+1}, \cdots, x_{n+K}\right)=Q_{f}, \theta\left(K+1, x_{n+1}, \cdots, x_{n+K}\right)=0, \partial \theta / \partial x_{n+i}(K+$ $\left.1, x_{n+1}, \cdots, x_{n+K}\right)=0$ and $\partial X / \partial x_{n+i}\left(K+1, x_{n+1}, \cdots, x_{n+K}\right)=O$ for $1 \leq i \leq$ $K$, we can resultant ODEs backwards in $\tau$ to find the values of $X, \theta$ and their derivatives with respect to $x_{n+i}$ over $\tau \in[0, K+1]$.

Remark 8. With Regard to Second-Order Derivatives: If we need the second order derivatives of $J\left(x_{n+1}\right)$ (e.g., refer to [21]) on the above nonlinear optimization algorithm, we can obtain the values by the following similar methods to the first order derivatives. In order to obtain the values of the second order derivatives of $J\left(x_{n+1}\right)$, we need the values of $\partial^{2} X / \partial x_{n+1}^{2}\left(\tau, x_{n+1}\right)$ and $\partial^{2} \theta / \partial x_{n+1}^{2}\left(\tau, x_{n+1}\right)$ over the whole time interval $\tau \in[0,2]$. By taking the first and second-order differentiations of (8)-(9) and (12)-(13) with respect to $x_{n+1}$, we can form a set of ODEs. Along with the terminal and boundary conditions $X\left(2, x_{n+1}\right)=Q_{f}, \theta\left(2, x_{n+1}\right)=0, \partial X / \partial x_{n+1}\left(2, x_{n+1}\right)=O$, $\partial \theta / \partial x_{n+1}\left(2, x_{n+1}\right)=O, \partial^{2} X / \partial x_{n+1}^{2}\left(2, x_{n+1}\right)=O$, and $\partial^{2} \theta / \partial x_{n+1}^{2}\left(2, x_{n+1}\right)=$ $O$, we can easily solve the set of ODEs for $X, \theta, \partial X / \partial x_{n+1}, \partial \theta / \partial x_{n+1}, \partial^{2} X / \partial x_{n+1}^{2}$, and $\partial^{2} \theta / \partial x_{n+1}^{2}$ and obtain the value of $d^{2} J\left(\tau, x_{n+1}\right) / d x_{n+1}^{2}$ at each $x_{n+1}$.

\section{Conclusion}

In this paper, we have studied the noncausal optimal timing and tracking problem for the switched systems. We have presented an optimization algorithm based on the time parametrization approach by X. Xu and P. J. Antsaklis([19, $22])$. In order to obtain the values of the gradients of the performance index, we need to do numerical integration and have assumed the appropriate smoothness 
on the reference signals. In spite of these task of numerical integration and assumption on the reference signals, we can expect better tracking performance and more appropriate switching timings by the noncausal information of the reference signals. Our noncausal tracking theory can be also applied to more general embedded systems. To clarify relationship between switched and more general embedded systems from the point of view of noncausal tracking control is a very important issue to research.

\section{References}

1. S-i. Azuma, Egerstedt and Y. Wardi: Output-Based Optimal Timing Control of Switched Systems, Lecture Notes in Computer Science, Vol. 3927, HSCC 2006, pp. 64-78, Springer-Verlag, 2006.

2. S. C. Bengea and R. A. DeCarlo: Optimal Control of Switching Systems, Automatica, Vol. 41, No.1, pp. 11-27, 2005.

3. D. P. Bertsekas: Nonlinear Programming, 2nd ed., Athena Scientific, 1999.

4. A. Cohen and U. Shaked: Linear Discrete-Time $\mathrm{H}_{\infty}$-Optimal Tracking with Preview, IEEE Trans. Automat. Contr., Vol. 42, pp. 270-276, 1997.

5. S. Devasia, D. Chen and B. Paden: Nonlinear Inversion-Based Output Tracking, IEEE Trans. Automat. Contr., Vol. 41, pp. 930-942, 1996.

6. M. Egerstedt, S-i. Azuma and Y. Wardi: Optimal Timing Control of Switched Linear Systems Based on Partial Information, Nonlinear Analysis, Vol. 65, pp. 1736-1750, 2006.

7. E. Gershon, D. J. N. Limebeer, U. Shaked and I. Yaesh: Stochastic $\mathrm{H}_{\infty}$ Tracking with Preview for State-Multiplicative Systems, IEEE Trans. Automat. Contr., Vol. 49, pp. 2061-2068, 2004.

8. E. Gershon, U. Shaked and I. Yaesh: $\mathrm{H}_{\infty}$ Tracking of Linear Continuous-Time Systems with Stochastic Uncertainties and Preview, Int. J. Robust and Nonlinear Control, Vol. 14, pp. 607-626, 2004.

9. E. Gershon, U. Shaked and I. Yaesh: $\mathrm{H}_{\infty}$ Control and Estimation of StateMultiplicative Linear Systems, Lecture Notes in Control and Information Sciences, Vol. 318, 2005.

10. A. Kojima and S. Ishijima: $\mathrm{H}_{\infty}$ performance of preview control systems, Automatica, Vol. 39, pp. 693-701, 2003.

11. A. Kojima and S. Ishijima: $H_{\infty}$ preview tracking in output feedback setting, Int. J. Robust and Nonlinear Control, Vol. 14, pp. 627-641, 2004.

12. A. Kojima and S. Ishijima: Formulas on Preview and Delayed $\mathrm{H}^{\infty}$ Control, IEEE Trans. Automat. Contr., Vol. 51, No. 12, pp. 1920-1937, 2006.

13. G. Nakura: $\mathrm{H}_{\infty}$ Tracking with Preview by State Feedback for Linear Jump Systems, Trans. of the Society of Instrument and Control Engneers (SICE), Vol. 42, No. 6, pp. 628-635, 2006 (in Japanese).

14. G. Nakura: Stochastic $\mathrm{H}_{\infty}$ Tracking with Preview by State Feedback for a Linear Jump System, 35th Symposium on Control Theory (in Japan), pp. 237-242, 2006.

15. G. Nakura: $\mathrm{H}_{\infty}$ Tracking with Preview for Linear Systems with Impulsive Effects -State Feedback and Full Information Cases-, accepted, to appear in the 17th IFAC World Congress, Seoul, 2008.

16. G. Nakura: $\mathrm{H}_{\infty}$ Tracking with Preview by Output Feedbak for Linear Systems with Impulsive Effects, accepted, to appear in the 17th IFAC World Congress, Seoul, 2008 . 
17. U. Shaked and C. E. de Souza: Continuous-Time Tracking Problems in an $\mathrm{H}_{\infty}$ Setting: A Game Theory Approach, IEEE Trans. Automat. Contr., Vol. 40, No. 5, pp. 841-852, 1995.

18. C. E. de Souza, U. Shaked and M. Fu: Robust $\mathrm{H}_{\infty}$-Tracking: A Game Theory Approach, Int. J. Robust and Nonlinear Control, Vol. 5, pp. 223-238, 1995.

19. X. Xu and P. J. Antsaklis: An Approach for Solving General Switched Linear Quadratic Optimal Control Problems, in Proc. 40th IEEE Conf. Decision Control, pp. 2478-2483, 2001.

20. X. Xu and P. J. Antsaklis: Optimal Control of Switched systems via Nonlinear Optimization Based on Direct Differentiations of Value Functions, Int. J. Contr., Vol. 75 , pp. 1406-1426, 2002

21. X. Xu and P.J. Antsaklis: Optimal Timing Control of a Class of Hybrid Autonomous Systems, International Journal of Hybrid Systems, Vol. 3, No. 1, pp. 33-60, 2003.

22. X. Xu and P. J. Antsaklis: Optimal Control of Switched Systems Based on Parameterization of the Switching Instants, IEEE Trans. Automat. Contr., Vol. 49, No. 1, pp. 2-16, 2004.

\section{Appendix: Proof of Proposition 1}

In this appendix, we give the proof of Proposition 1. This is the modification of the preview tracking control theory by U. Shaked and C. E. de Souza([17]) for the noncausal GSLQ tracking problem.

\section{(Proof of Proposition 1)}

Sufficiency: For $\tau \in[0,1)$, not considering any preview or noncausal information, we can easily show

$$
\begin{aligned}
& \quad \frac{d}{d \tau}\left\{x^{\prime}\left(\tau, x_{n+1}\right) P\left(\tau, x_{n+1}\right) x\left(\tau, x_{n+1}\right)\right\} \\
& =\left(x_{n+1}-t_{0}\right)\left\{-\left\|z_{c}\left(\tau, x_{n+1}\right)\right\|^{2}\right. \\
& +\left\|u\left(\tau, x_{n+1}\right)+\tilde{R}^{-1} \tilde{S}_{1} x\left(\tau, x_{n+1}\right)+\mathbf{C}_{u} r_{c}\left(\tau, x_{n+1}\right)\right\|_{\tilde{R}}^{2} \\
& \left.\quad-2 x^{\prime}\left(\tau, x_{n+1}\right) \bar{B}_{c, 1}\left(\tau, x_{n+1}\right) r_{c}\left(\tau, x_{n+1}\right)+\delta J_{c}\left(r_{c}, \tau, x_{n+1}\right)\right\}
\end{aligned}
$$

where we have used the general Riccati equation (8)-(9). Now introducing the vector $\theta$, which can include some preview information of the tracking signals,

$$
\begin{aligned}
& \frac{d}{d \tau}\left\{\theta^{\prime}\left(\tau, x_{n+1}\right) x\left(\tau, x_{n+1}\right)\right\} \\
= & \frac{\partial}{\partial \tau} \theta^{\prime}\left(\tau, x_{n+1}\right) x\left(\tau, x_{n+1}\right) \\
& +\left(x_{n+1}-t_{0}\right) \theta^{\prime}\left(\tau, x_{n+1}\right)\left(\bar{A}_{c, 1} x\left(\tau, x_{n+1}\right)+B_{2,1} \hat{u}_{c, 1}\left(\tau, x_{n+1}\right)+B_{3,1} r_{c}\left(\tau, x_{n+1}\right)\right)
\end{aligned}
$$

where $\hat{u}_{c, 1}\left(\tau, x_{n+1}\right)=u\left(\tau, x_{n+1}\right)+\tilde{R}^{-1} \tilde{S}_{1} x\left(\tau, x_{n+1}\right)$. As a result,

$$
\begin{aligned}
& \frac{d}{d \tau}\left\{x^{\prime}\left(\tau, x_{n+1}\right) X\left(\tau, x_{n+1}\right) x\left(\tau, x_{n+1}\right)\right\}+2 \frac{d}{d \tau}\left\{\theta^{\prime}\left(\tau, x_{n+1}\right) x\left(\tau, x_{n+1}\right)\right\} \\
&=\left(x_{n+1}-t_{0}\right)\left\{-\| C_{1} x\left(\tau, x_{n+1}\right)+D_{12} u(\tau,\right.\left.x_{n+1}\right)+D_{13} r_{c}\left(\tau, x_{n+1}\right) \|^{2} \\
&+\left\|\hat{u}_{c, 1}\left(\tau, x_{n+1}\right)+\mathbf{C}_{u} r_{c}\left(\tau, x_{n+1}\right)+\mathbf{C}_{\theta u, 1} \theta\left(\tau, x_{n+1}\right)\right\|_{\tilde{R}}^{2} \\
&\left.+\delta \bar{J}_{c, 1}\left(r_{c}, \tau, x_{n+1}\right)\right\}
\end{aligned}
$$


where we have used the dynamics (12). Similarly, for $\tau \in[1,2]$, using the dynamics (13), we obtain

$$
\begin{gathered}
\frac{d}{d \tau}\left\{x^{\prime}\left(\tau, x_{n+1}\right) X\left(\tau, x_{n+1}\right) x\left(\tau, x_{n+1}\right)\right\}+2 \frac{d}{d \tau}\left\{\theta^{\prime}\left(\tau, x_{n+1}\right) x\left(\tau, x_{n+1}\right)\right\} \\
=\left(T-x_{n+1}\right)\left\{-\left\|C_{1} x\left(\tau, x_{n+1}\right)+D_{12} u\left(\tau, x_{n+1}\right)+D_{13} r_{c}\left(\tau, x_{n+1}\right)\right\|^{2}\right. \\
+\left\|\hat{u}_{c, 2}\left(\tau, x_{n+1}\right)+\mathbf{C}_{u} r_{c}\left(\tau, x_{n+1}\right)+\mathbf{C}_{\theta u, 2} \theta\left(\tau, x_{n+1}\right)\right\|_{\tilde{R}}^{2} \\
\left.+\delta \bar{J}_{c, 2}\left(r_{c}, \tau, x_{n+1}\right)\right\}
\end{gathered}
$$

where $\hat{u}_{c, 2}\left(\tau, x_{n+1}\right)=u\left(\tau, x_{n+1}\right)+\tilde{R}^{-1} \tilde{S}_{2} x\left(\tau, x_{n+1}\right)$. Integrating (23) and (24) from $\tau=0$ to $\tau=2$ piecewise, we obtain

$$
\begin{gathered}
x^{\prime}\left(2, x_{n+1}\right) P\left(2, x_{n+1}\right) x\left(2, x_{n+1}\right)-x^{\prime}\left(0, x_{n+1}\right) P\left(0, x_{n+1}\right) x\left(0, x_{n+1}\right) \\
+2 \theta^{\prime}\left(2, x_{n+1}\right) x\left(2, x_{n+1}\right)-2 \theta^{\prime}\left(0, x_{n+1}\right) x\left(0, x_{n+1}\right) \\
=\left(x_{n+1}-t_{0}\right) \int_{0}^{1}\left\{-\left\|z_{c}\left(\tau, x_{n+1}\right)\right\|^{2}+\delta \bar{J}_{c, 1}\left(r_{c}, \tau, x_{n+1}\right)\right. \\
\left.+\left\|\hat{u}_{c, 1}\left(\tau, x_{n+1}\right)+\mathbf{C}_{u} r_{c}\left(\tau, x_{n+1}\right)+\mathbf{C}_{\theta u, 1} \theta\left(\tau, x_{n+1}\right)\right\|_{\tilde{R}}^{2}\right\} d \tau \\
+\left(T-x_{n+1}\right) \int_{1}^{2}\left\{-\left\|z_{c}\left(\tau, x_{n+1}\right)\right\|^{2}+\delta \bar{J}_{c, 2}\left(r_{c}, \tau, x_{n+1}\right)\right. \\
\left.+\left\|\hat{u}_{c, 2}\left(\tau, x_{n+1}\right)+\mathbf{C}_{u} r_{c}\left(\tau, x_{n+1}\right)+\mathbf{C}_{\theta u, 2} \theta\left(\tau, x_{n+1}\right)\right\|_{\tilde{R}}^{2}\right\} d \tau .
\end{gathered}
$$

Including the noncausal part $\theta\left(\tau, x_{n+1}\right)$ at time $\tau$, we adopt

$$
\begin{aligned}
& \hat{u}_{c, 1}^{*}\left(\tau, x_{n+1}\right)=-\mathbf{C}_{u} r_{c}\left(\tau, x_{n+1}\right)-\mathbf{C}_{\theta u, 1} \theta\left(\tau, x_{n+1}\right) \text { for } \tau \in[0,1), \\
& \hat{u}_{c, 2}^{*}\left(\tau, x_{n+1}\right)=-\mathbf{C}_{u} r_{c}\left(\tau, x_{n+1}\right)-\mathbf{C}_{\theta u, 2} \theta\left(\tau, x_{n+1}\right) \text { for } \tau \in[1,2]
\end{aligned}
$$

as the optimal control strategy. By the terminal conditions $P\left(2, x_{n+1}\right)=Q_{T}$ and $\theta\left(2, x_{n+1}\right)=0$, we get the result.

Necessity: Because of arbitrality of the reference signal $r_{c}(\cdot)$, by considering the case of $r_{c}(\cdot) \equiv 0$, one can easily deduce the necessity for the solvability of our GSLQ tracking problem. For the purpose, one can get the parametrized general Hamiltion-Jacobi equation by applying the standard dynamic programming method based on the principle of optimality. We can obtain the parametrized general Riccati equation (8)-(9) by restricting the form of the value function to be quadratic as follows:

$$
V\left(x, \tau, x_{n+1}\right)=x^{\prime} X\left(\tau, x_{n+1}\right) x
$$

(QED.) 